\title{
An extremal problem in Banach algebras
}

\author{
by \\ Anders Olofsson (Stockholm)
}

\begin{abstract}
We study asymptotics of a class of extremal problems $r_{n}(A, \varepsilon)$ related to norm controlled inversion in Banach algebras. In a general setting we prove estimates that can be considered as quantitative refinements of a theorem of Jan-Erik Björk [1]. In the last section we specialize further and consider a class of analytic Beurling algebras. In particular, a question raised by Jan-Erik Björk in [1] is answered in the negative.
\end{abstract}

1. Introduction. Let $A$ be a (unitary) topological Banach algebra (see below) with norm $\|\cdot\|$ and denote by $r(f)$ the spectral radius of $f \in A$. In this note we study certain aspects of the extremal problem

$$
r_{n}(A, \varepsilon)=\sup \left\{\left\|f^{n}\right\|: f \in A,\|f\| \leq 1, r(f) \leq \varepsilon\right\} \quad(n \geq 1,0<\varepsilon<1)
$$

In particular, we are interested in the limit behavior of (1) as $n \rightarrow \infty$. One motivation for the study of this extremal problem is its connection to norm controlled inversion in Banach algebras (see [1], [2], [3], [4] and [6]). (1) can also be found in [5].

In Section 2 we give asymptotic upper bounds for $r_{n}(A, \varepsilon)$ and the related quantity $r_{n}(A)$ (see Definition 1, Remark 1 and Proposition 1) introduced by Jan-Erik Björk in [1]. The main results in this section are Theorem 1, Corollary 1 and Theorem 2. In Theorem 1 we give a universal upper bound for the quantity $\lim _{n \rightarrow \infty} r_{n}(A, \varepsilon)^{1 / n}$ (for the existence of the limit see Remark 1). In Corollary 1 a corresponding estimate for the quantities $r_{n}(A)$ is given. Our Theorem 1 and Corollary 1 are quantitative refinements of a theorem of J.-E. Björk in [1] (Theorem 3.1). (See also Remark 2.) In Theorem 2 we give an estimate of $\lim _{n \rightarrow \infty} r_{n}(A, \varepsilon)^{1 / n}$ in terms of the quantity $\delta_{1}(A)$ (Definition 2), connected with a certain quantitative form of Wiener's lemma previously studied in [6], [3], [2] and [4]. The corresponding estimate for $\lim _{n \rightarrow \infty} r_{n}(A)$ is also given. Theorem 2 is an extension of Théorème 3.1 in [2] and has a similar proof. The proofs of these results are completely elementary.

2000 Mathematics Subject Classification: Primary 46J05; Secondary 42A20.

Key words and phrases: norm controlled inversion, Wiener's lemma, Beurling algebras. 
The material in Section 3 is inspired by [3] and [2]. We consider analytic Beurling algebras $A_{\omega}^{+}$corresponding to Banach algebra weights $\omega$ on $\mathbb{N}$ (see below) such that $\omega(n) \rightarrow c \in[1, \infty)$ as $n \rightarrow \infty$. Of course, these algebras are just certain renormings of the well known Wiener algebra $A^{+}$of absolutely convergent Taylor series in the unit disc $\mathbb{D}$. In Theorem 3 we compute the quantities $r_{n}\left(A_{\omega}^{+}\right)$and $K_{0}\left(A_{\omega}^{+}\right)$(see Corollary 1) for such an algebra $A_{\omega}^{+}$. Theorem 3 exemplifies a sensitivity of the numbers $r_{n}(A)$ and the problem of norm controlled inversion for the particular choice of norm in $A$. In particular, Theorem 3 answers the following question raised in [1] (page 284, line 1): For a commutative semisimple Banach algebra $A$, does $r_{n}(A)<1$ for some $n \geq 2$ imply $r_{n}(A) \rightarrow 0$ ? In fact, under these circumstances, the limit $\lim _{n \rightarrow \infty} r_{n}(A)$ exists and can be any number in the half-open interval $[0,1)$. Examples are provided by suitable algebras $A_{\omega}^{+}$. (See also Remarks 5 and 6.) The proof of Theorem 3 uses ideas from a construction of Y. Katznelson presented in [7] combined with a recent lemma of O. El-Fallah (Lemma 2 in Section 3 below).

Acknowledgements. The author is grateful to Jan-Erik Björk of Stockholm University for his valuable suggestions regarding this manuscript. The author also wants to thank Mats Erik Andersson of Stockholm University for his careful reading of the manuscript. The author is also indebted to Anders Dahlner of Lund University for many valuable discussions on the subject of the present article.

Topological Banach algebras. By a topological Banach algebra we mean a Banach space $A$ equipped with a multiplication, continuous as a map $A \times A \rightarrow A$, in such a way that $A$ becomes a commutative complex algebra with unit. The unit element of $A$ is denoted by $e$. We assume that the norm of $A$ is normalized by $\|e\|=1$. The continuity of the multiplication can equivalently be formulated by saying that the inequality

$$
\|f g\| \leq C\|f\| \cdot\|g\|, \quad f, g \in A,
$$

holds for some constant $C \in[1, \infty)$. As is well known, every topological Banach algebra becomes a Banach algebra after a suitable renorming (passage to operator norm). In a topological Banach algebra $A$ the spectral radius formula holds in the ordinary sense, i.e.,

$$
r(f)=\|\widehat{f}\|_{\infty}=\lim _{n \rightarrow \infty}\left\|f^{n}\right\|^{1 / n}, \quad f \in A,
$$

where $\widehat{f}$ denotes the Gelfand transform of $f$ and $\|\cdot\|_{\infty}$ is the maximum norm on the maximal ideal space of $A$. The validity of (3) is clear since either side of (3) is unaffected by a renorming of $A$. 
Beurling algebras. By a Banach algebra weight $\omega$ on $\mathbb{N}=\{0,1,2, \ldots\}$ we mean a positive weight function $\omega$ such that

$$
\omega(0)=1, \quad \omega(n+m) \leq \omega(n) \omega(m), \quad n, m \geq 0 .
$$

The corresponding analytic Beurling algebra normed by

$$
\|f\|_{\omega}=\sum_{k=0}^{\infty}\left|a_{k}\right| \omega(k), \quad f=\sum_{k=0}^{\infty} a_{k} z^{k}
$$

is denoted by $A_{\omega}^{+}$. For $\omega \equiv 1$ we write $A^{+}=A_{\omega}^{+}$and $\|\cdot\|=\|\cdot\|_{\omega}$. Most weights in this note are such that $\omega(k)^{1 / k} \rightarrow 1$. By this normalization the maximal ideal space of $A_{\omega}^{+}$is canonically identified with the closed unit disc $\overline{\mathbb{D}}$ and $r(f)=\|f\|_{\infty}$, where $\|\cdot\|_{\infty}$ denotes the maximum norm on $\overline{\mathbb{D}}$.

2. Topological Banach algebras. In the proof of Theorem 1 we use the following lemma:

Lemma 1. Let $A$ be a topological Banach algebra. For $f \in A$ with $r(f)<1$, the following identity holds:

$$
\left(\begin{array}{c}
n+k \\
k
\end{array}\right) f^{n}=\frac{1}{2 \pi} \int_{\mathbb{T}} e^{-i n \theta}\left(e-e^{i \theta} f\right)^{-k-1} d \theta, \quad n \geq 1, k \geq 0 .
$$

Proof. We have the power series expansion

$$
\frac{1}{(1-z)^{k+1}}=\sum_{n=0}^{\infty}\left(\begin{array}{c}
n+k \\
k
\end{array}\right) z^{n} .
$$

Substituting $z=e^{i \theta} f$ and integrating, we obtain the lemma.

Theorem 1. Assume that the topological Banach algebra A satisfies a bounded inverse formula in the sense that there exist constants $\varepsilon \in(0,1)$ and $K=K(\varepsilon) \in[1, \infty)$ such that

$$
\left\|(e-f)^{-1}\right\| \leq K \quad \text { if }\|f\| \leq 1 \text { and } r(f) \leq \varepsilon .
$$

Then

$$
\lim _{n \rightarrow \infty} r_{n}(A, \varepsilon)^{1 / n} \leq 1-\frac{1}{C K},
$$

where $C \geq 1$ is given by (2). (For the existence of the limit see Remark 1.)

Proof. By Lemma 1 and (4) we have

$$
\left(\begin{array}{c}
n+k \\
n
\end{array}\right) r_{n}(A, \varepsilon) \leq C^{k} K^{k+1}, \quad n \geq 1, k \geq 0 .
$$

Using Stirling's formula one verifies that, for $c \in(0, \infty)$,

$$
\lim _{\substack{n \rightarrow \infty \\
|k / n-c| \leq 1 / n}}\left(\begin{array}{c}
n+k \\
n
\end{array}\right)^{1 / n}=\frac{(1+c)^{1+c}}{c^{c}} .
$$


In the limit as $n \rightarrow \infty,|k / n-c| \leq 1 / n$, we deduce from (6) that

$$
\lim _{n \rightarrow \infty} r_{n}(A, \varepsilon)^{1 / n} \leq \frac{1}{1+c}\left(\frac{c}{1+c}\right)^{c} C^{c} K^{c} .
$$

Choosing $c=1 /(C K-1)$ in this inequality yields $(5)$.

Definition 1 (J.-E. Björk [1]). Let $A$ be a topological Banach algebra. A sequence $\left\{f_{j}\right\}$ in $A$ is called a spectral null sequence if $\left\|f_{j}\right\| \leq 1$ and $r\left(f_{j}\right) \rightarrow 0$. If $\left\{f_{j}\right\}$ is a spectral null sequence and $n \geq 1$, then $r_{n}\left(\left\{f_{j}\right\}\right)$ is defined by $r_{n}\left(\left\{f_{j}\right\}\right)=\lim \sup _{j \rightarrow \infty}\left\|f_{j}^{n}\right\|^{1 / n}$. The number $r_{n}(A)$ is defined by $r_{n}(A)=\sup r_{n}\left(\left\{f_{j}\right\}\right)$, where the supremum is taken over all spectral null sequences $\left\{f_{j}\right\}$ in $A$.

REMARK 1. It is immediate from the definition of the numbers $r_{n}(A)$ that

$$
r_{n+m}(A)^{n+m} \leq C r_{n}(A)^{n} r_{m}(A)^{m}, \quad n, m \geq 1,
$$

where $C$ is given by (2). By this submultiplicativity type inequality, $\lim _{n \rightarrow \infty} r_{n}(A)$ exists. The same argument establishes the existence of $\lim _{n \rightarrow \infty} r_{n}(A, \varepsilon)^{1 / n}$.

The relation between $r_{n}(A)$ and the extremal problem (1) is given by the following proposition:

Proposition 1. In a topological Banach algebra $A$ the following holds:

$$
\lim _{\varepsilon \rightarrow 0} r_{n}(A, \varepsilon)^{1 / n}=r_{n}(A) .
$$

Proof. Let $\left\{f_{j}\right\}$ be a spectral null sequence in $A$. For $j$ large we have $\left\|f_{j}^{n}\right\| \leq r_{n}(A, \varepsilon)$. Taking limits and suprema we get $r_{n}(A) \leq$ $\lim _{\varepsilon \rightarrow 0} r_{n}(A, \varepsilon)^{1 / n}$.

Let $\delta>0$. It is easily seen that there exists an $\varepsilon>0$ such that $\left\|f^{n}\right\|^{1 / n} \leq$ $r_{n}(A)+\delta$ if $\|f\| \leq 1$ and $r(f) \leq \varepsilon$. From this we have $\lim _{\varepsilon \rightarrow 0} r_{n}(A, \varepsilon)^{1 / n} \leq$ $r_{n}(A)$.

Corollary 1. Let $A$ be a topological Banach algebra. For $\varepsilon>0$ write

$$
\begin{aligned}
K(\varepsilon, A) & =\sup \left\{\left\|(e-f)^{-1}\right\|: f \in A,\|f\| \leq 1, r(f) \leq \varepsilon\right\}, \\
K_{0} & =K_{0}(A)=\lim _{\varepsilon \rightarrow 0} K(\varepsilon, A) .
\end{aligned}
$$

Then

$$
\lim _{n \rightarrow \infty} r_{n}(A) \leq 1-\frac{1}{C K_{0}}
$$

where $C$ is given by (2).

Proof. By Proposition 1, the corollary follows from Theorem 1 upon letting $\varepsilon \rightarrow 0$. 
Remark 2. There is an obvious converse to Theorem 1 and Corollary 1. Assume (5) holds. For $f \in A,\|f\| \leq 1, r(f) \leq \varepsilon$, we have

$$
\left\|(e-f)^{-1}\right\|=\left\|\sum_{n=0}^{\infty} f^{n}\right\| \leq \sum_{n=0}^{\infty} r_{n}(A, \varepsilon)<\infty .
$$

Moreover, for any function $g=\sum a_{n} z^{n}$ analytic in an open set containing $(1-1 /(C K)) \overline{\mathbb{D}}$, we have

$$
\|g(f)\| \leq \sum_{n=0}^{\infty}\left|a_{n}\right| r_{n}(A, \varepsilon)<\infty,
$$

whenever $\|f\| \leq 1$ and $r(f) \leq \varepsilon$.

In [6], [7], [3], [2] and [4] a somewhat different quantitative Wiener lemma, than the possibility of (4) to hold, is studied. Indeed, given $\|f\| \leq 1$ and $|\widehat{f}| \geq \delta>0$, one wants to estimate $\left\|f^{-1}\right\|$. ( $\widehat{f}$ denotes the Gelfand transform of $f$.) This is formalized in the following definition.

Definition (N. K. Nikolski $[3,4])$. Let $A$ be a topological Banach algebra. For $0<\delta \leq 1$ we define

$$
\begin{aligned}
c_{1}(A, \delta) & =\sup \{\|1 / f\|: f \in A,\|f\| \leq 1,|\widehat{f}| \geq \delta\}, \\
\delta_{1}(A) & =\inf \left\{\delta \in(0,1]: c_{1}(A, \delta)<\infty\right\} .
\end{aligned}
$$

(We use the convention that $\inf \emptyset=\infty$.)

The following theorem is an extension of Théorème 3.1 of [2].

Theorem 2. Let $A$ be a topological Banach algebra and define

$$
r(A)=\lim _{n \rightarrow \infty} r_{n}(A) .
$$

Assume $\delta_{1}(A)<1$. Then

$$
\begin{gathered}
\lim _{n \rightarrow \infty} r_{n}(A, \varepsilon)^{1 / n} \leq \frac{\varepsilon+\delta_{1}(A)}{1-\delta_{1}(A)}, \\
r(A) \leq \frac{\delta_{1}(A)}{1-\delta_{1}(A)}, \quad \frac{r(A)}{1+r(A)} \leq \delta_{1}(A) .
\end{gathered}
$$

Proof. It is straightforward to check that $r(A) \leq \delta_{1}(A) /\left(1-\delta_{1}(A)\right)$ follows from (8) by letting $\varepsilon \rightarrow 0$ and that the two inequalities in (9) are equivalent. Hence, it suffices to prove (8).

Let $f \in A,\|f\| \leq 1$ and $r(f) \leq \varepsilon$. Let $z \in \mathbb{C}$. Since the element $(e-z f) /(1+|z|)$ is of norm $\leq 1$ and has Gelfand transform of minimal modulus $\geq(1-|z| \varepsilon) /(1+|z|)$, we have

$$
(1+|z|)\left\|(e-z f)^{-1}\right\| \leq c_{1}\left(A, \frac{1-|z| \varepsilon}{1+|z|}\right)<\infty \quad \text { provided } \frac{1-|z| \varepsilon}{1+|z|}>\delta_{1}(A) .
$$


Write $z=r e^{i \theta}$ and assume $r>0$ is such that $(1-r \varepsilon) /(1+r)>\delta_{1}(A)$. Since $r \varepsilon<1$, we have $\left(e-r e^{i \theta} f\right)^{-1}=\sum_{n=0}^{\infty} e^{i n \theta} r^{n} f^{n}$ in $A$, and

$$
r^{n} f^{n}=\frac{1}{2 \pi} \int_{\mathbb{T}} e^{-i n \theta}\left(e-r e^{i \theta} f\right)^{-1} d \theta .
$$

By an obvious estimate we have

$$
r^{n}\left\|f^{n}\right\| \leq \frac{1}{1+r} c_{1}\left(A, \frac{1-r \varepsilon}{1+r}\right)
$$

so that

$$
r^{n} r_{n}(A, \varepsilon) \leq \frac{1}{1+r} c_{1}\left(A, \frac{1-r \varepsilon}{1+r}\right) .
$$

Taking the $n$th roots and passing to the limit we obtain

$$
\lim _{n \rightarrow \infty} r_{n}(A, \varepsilon)^{1 / n} \leq 1 / r \quad \text { if } \frac{1-r \varepsilon}{1+r}>\delta_{1}(A) .
$$

Letting $(1-r \varepsilon) /(1+r) \rightarrow \delta_{1}(A)$ yields $(8)$.

REMARK 3. In all cases known to the author, equality holds in (9).

3. Analytic Beurling algebras. In the present section $\|\cdot\|$ always denotes the norm of absolutely convergent Taylor series on $\mathbb{D}$ (see Section 1). We begin with some preliminary lemmas needed in the proof of Theorem 3 .

Definition 3 (O. El-Fallah [2]). Let $\omega$ be a Banach algebra weight on $\mathbb{N}$. For positive integers $n, k$ the following quantities are considered:

$$
\begin{aligned}
a(k, n, \omega) & =\sup \left\{\left(\frac{\omega\left(m_{1}+\ldots+m_{n}\right)}{\omega\left(m_{1}\right) \ldots \omega\left(m_{n}\right)}\right)^{1 / n}: m_{j} \geq k, j=1, \ldots, n\right\}, \\
a(n, \omega) & =\lim _{k \rightarrow \infty} a(k, n, \omega) .
\end{aligned}
$$

Lemma 2 (O. El-Fallah [2], Lemme 5.3). Let $\omega$ and $a$ be as in Definition 3. Assume $\omega(k)^{1 / k} \rightarrow 1$ as $k \rightarrow \infty$. Then, for $f \in A_{\omega}^{+}$with $\|f\|_{\omega} \leq 1$, the following inequality holds:

$$
\left\|f^{n}\right\|_{\omega} \leq r(f) n \sum_{m=0}^{k-1} \omega(m)+a(k, n, \omega)^{n} .
$$

In particular, $r_{n}\left(A_{\omega}^{+}\right) \leq a(n, \omega)$.

Proof. Since

$$
\begin{aligned}
f^{n}= & \left(\sum_{m=0}^{k-1} a_{m} z^{m}\right)\left[f^{n-1}+f^{n-2}\left(\sum_{m=k}^{\infty} a_{m} z^{m}\right)+\ldots\right. \\
& \left.+f\left(\sum_{m=k}^{\infty} a_{m} z^{m}\right)^{n-2}+\left(\sum_{m=k}^{\infty} a_{m} z^{m}\right)^{n-1}\right]+\left(\sum_{m=k}^{\infty} a_{m} z^{m}\right)^{n}
\end{aligned}
$$


we have

$$
\left\|f^{n}\right\|_{\omega} \leq n\left\|\sum_{m=0}^{k-1} a_{m} z^{m}\right\|_{\omega}+\left\|\left(\sum_{m=k}^{\infty} a_{m} z^{m}\right)^{n}\right\|_{\omega} .
$$

The first term is estimated by

$$
\left\|\sum_{m=0}^{k-1} a_{m} z^{m}\right\|_{\omega} \leq r(f) \sum_{m=0}^{k-1} \omega(m)
$$

and the last term is estimated by

$$
\left\|\left(\sum_{m=k}^{\infty} a_{m} z^{m}\right)^{n}\right\|_{\omega} \leq \sum_{m_{j} \geq k}\left|a_{m_{1}}\right| \ldots\left|a_{m_{n}}\right| \omega\left(m_{1}+\ldots+m_{n}\right) \leq a(k, n, \omega)^{n}
$$

LEMMA 3. Let $n$ be a positive integer and $\varepsilon>0$. Then there exists $f \in$ $A^{+}$with $\|f\|=\left\|f^{2}\right\|=\ldots=\left\|f^{n}\right\|=1$ and $\|f\|_{\infty}<\varepsilon$. (In fact, $f$ can be chosen to be a polynomial.) In particular, $r_{n}\left(A^{+}, \varepsilon\right)=1$ for all $n \geq 1$ and $\varepsilon \in(0,1)$.

Proof. Let $g(z)=(1+z)\left(1-z^{n+1}\right) / 4$. Now, $g$ is a polynomial with $\|g\|=$ $\left\|g^{2}\right\|=\ldots=\left\|g^{n}\right\|=1$ and $\|g\|_{\infty}<1$. Setting $f(z)=g(z) g\left(z^{n_{1}}\right) \ldots g\left(z^{n_{r}}\right)$ for some $1 \ll n_{1} \ll \ldots \ll n_{r}$ we achieve $\|f\|=\left\|f^{2}\right\|=\ldots=\left\|f^{n}\right\|=1$ and $\|f\|_{\infty}<\varepsilon$.

REMARK 4. In the above form, Lemma 3 is due to H. S. Shapiro and G. Ryd, and has been communicated to the author by A. Dahlner. In [7] a somewhat weaker version of Lemma 3 was used.

The following lemma is well known.

Lemma 4. Let $f_{k} \in A^{+}, k=0,1, \ldots$, be such that $\sum\left\|f_{k}\right\|<\infty$. Then

$$
\lim _{N \rightarrow \infty}\left\|\sum_{k=0}^{\infty} z^{k N} f_{k}\right\|=\sum_{k=0}^{\infty}\left\|f_{k}\right\|
$$

THEOREM 3. Let $\omega$ be a Banach algebra weight on $\mathbb{N}$ such that $\omega(k) \rightarrow$ $c \in[1, \infty)$ as $k \rightarrow \infty$. Then, for the corresponding analytic Beurling algebra $A_{\omega}^{+}$, the following holds:

$$
\begin{aligned}
r_{n}\left(A_{\omega}^{+}\right) & =a(n, \omega)=c^{1 / n-1} \quad \text { for } n \geq 1, \\
K_{0}\left(A_{\omega}^{+}\right): & =\lim _{\varepsilon \rightarrow 0} K\left(\varepsilon, A_{\omega}^{+}\right)=1+c /(c-1)=(2 c-1) /(c-1) .
\end{aligned}
$$

For $c=1$ the right hand side of (11) is to be interpreted as $+\infty$.

In (11) we have written

$$
K\left(\varepsilon, A_{\omega}^{+}\right)=\sup \left\{\left\|\frac{1}{1-f}\right\|_{\omega}:\|f\|_{\omega} \leq 1, r(f) \leq \varepsilon\right\} .
$$

In the proof below this quantity is denoted by $K(\varepsilon)$. 
Proof. We first prove (10). Since

$$
\frac{\omega\left(m_{1}+\ldots+m_{n}\right)}{\omega\left(m_{1}\right) \ldots \omega\left(m_{n}\right)} \rightarrow \frac{c}{c^{n}}
$$

as $m_{j} \rightarrow \infty$, by Lemma 2 we have $r_{n}\left(A_{\omega}^{+}\right)^{n} \leq a(n, \omega)^{n}=c / c^{n}$. Next we prove $r_{n}\left(A_{\omega}^{+}\right)^{n} \geq c / c^{n}$. By Lemma 3 we can choose a sequence $\left\{f_{j}\right\} \subset A^{+}$ such that

$$
\left\|f_{j}\right\|=\ldots=\left\|f_{j}^{j}\right\|=1 \quad \text { and } \quad r\left(f_{j}\right) \rightarrow 0 .
$$

Now $\left\|\left(f_{j} /\left\|f_{j}\right\|_{\omega}\right)\right\|_{\omega}=1$ and $r\left(f_{j} /\left\|f_{j}\right\|_{\omega}\right) \rightarrow 0$, whence

$$
\limsup _{j \rightarrow \infty}\left\|\left(f_{j} /\left\|f_{j}\right\|_{\omega}\right)^{n}\right\|_{\omega} \leq r_{n}\left(A_{\omega}^{+}\right)^{n} .
$$

Observe that $\left\|f_{j}^{n}\right\|_{\omega} \rightarrow c$ as $j \rightarrow \infty$. Since

$$
\left\|\left(f_{j} /\left\|f_{j}\right\|_{\omega}\right)^{n}\right\|_{\omega}=\frac{1}{\left\|f_{j}\right\|_{\omega}^{n}}\left\|f_{j}^{n}\right\|_{\omega} \rightarrow \frac{c}{c^{n}} \quad \text { as } j \rightarrow \infty,
$$

we have $r_{n}\left(A_{\omega}^{+}\right)^{n} \geq c / c^{n}$.

Next we prove (11). Let $\|f\|_{\omega} \leq 1, r(f) \leq \varepsilon$. Since

$$
\frac{1}{1-f}=\sum_{k=0}^{\infty} f^{k}
$$

we have

$$
\left\|\frac{1}{1-f}\right\|_{\omega} \leq \sum_{k=0}^{\infty}\left\|f^{k}\right\|_{\omega} \leq 1+\sum_{k=1}^{\infty} r_{k}\left(A_{\omega}^{+}, \varepsilon\right)
$$

Hence

$$
K(\varepsilon) \leq 1+\sum_{k=1}^{\infty} r_{k}\left(A_{\omega}^{+}, \varepsilon\right)
$$

Passing to the limit as $\varepsilon \rightarrow 0$, using (10) and Proposition 1 , we get $K_{0} \leq$ $1+\sum_{k=1}^{\infty} r_{k}\left(A_{\omega}^{+}\right)^{k}=(2 c-1) /(c-1)$.

Now we prove $K_{0} \geq(2 c-1) /(c-1)$. Let $\left\{f_{j}\right\} \subset A^{+}$be a sequence satisfying (12). For $j$ large we have

$$
K(\varepsilon) \geq\left\|\left(1-\frac{z^{N} f_{j}}{\left\|z^{N} f_{j}\right\|_{\omega}}\right)^{-1}\right\|_{\omega}=\left\|\sum_{k=0}^{\infty} \frac{1}{\left\|z^{N} f_{j}\right\|_{\omega}^{k}} z^{k N} f_{j}^{k}\right\|_{\omega} .
$$

Next we compute the limit as $N \rightarrow \infty$ of the right hand side in this inequality. Since

$$
\sum_{k=0}^{\infty}\left(\frac{1}{\left\|z^{N} f_{j}\right\|_{\omega}^{k}}-\frac{1}{c^{k}}\right) z^{k N} f_{j}^{k} \rightarrow 0 \quad \text { in } A_{\omega}^{+}, \quad N \rightarrow \infty,
$$


we have

$$
\left\|\sum_{k=0}^{\infty} \frac{1}{\left\|z^{N} f_{j}^{k}\right\|_{\omega}^{k}} z^{k N} f_{j}^{k}\right\|_{\omega}=\left\|\sum_{k=0}^{\infty} z^{k N}\left(f_{j} / c\right)^{k}\right\|_{\omega}+o(1) .
$$

Now

$$
\left\|\sum_{k=0}^{\infty} z^{k N}\left(f_{j} / c\right)^{k}\right\|_{\omega}=1+c\left\|\sum_{k=1}^{\infty} z^{k N}\left(f_{j} / c\right)^{k}\right\|+o(1)=1+c \sum_{k=1}^{\infty} \frac{1}{c^{k}}\left\|f_{j}^{k}\right\|+o(1),
$$

where in the last equality we have used Lemma 4. Summing up, we have shown

$$
K(\varepsilon) \geq 1+c \sum_{k=1}^{\infty} \frac{1}{c^{k}}\left\|f_{j}^{k}\right\|
$$

Letting $j \rightarrow \infty$ we get $K(\varepsilon) \geq 1+c \sum_{k=1}^{\infty} 1 / c^{k}=(2 c-1) /(c-1)$. From this (11) follows.

REMARK 5. In [1] (page 283, last paragraph), one more question besides the one alluded to in the introduction is asked. Namely, for a unitary commutative semi-simple Banach algebra $A$, does $r_{n}(A)<1$ for some $n>2$ imply $r_{2}(A)<1$ ? Recently, in [2] (Remarque 5.7), O. El-Fallah has, for given $m \geq 2$, constructed a weighted analytic Beurling algebra $A_{\omega}^{+}$with $r_{1}\left(A_{\omega}^{+}\right)=r_{2}\left(A_{\omega}^{+}\right)=\ldots=r_{m}\left(A_{\omega}^{+}\right)=1$ and $r_{n}\left(A_{\omega}^{+}\right)=0$ for $n>m$.

REMARK 6. Let $A$ be a commutative semisimple Banach algebra with unit element. Regarding the quantity $\lim _{n \rightarrow \infty} r_{n}(A)$ there is an amount of slack between the upper bound in Corollary 1 and the examples in Theorem 3 . The right upper bound for the quantity $\lim _{n \rightarrow \infty} r_{n}(A)$ remains to be found.

\section{References}

[1] J.-E. Björk, On the spectral radius formula in Banach algebras, Pacific J. Math. 40 (1972), 279-284.

[2] O. El-Fallah, Majorations uniformes de normes d'inverses dans les algèbres de Beurling, preprint, Département de Mathématiques et Informatique, Faculté des Sciences, Université Mohammed V, Rabat, Maroc, 1999.

[3] O. El-Fallah, N. K. Nikolski and M. Zarrabi, Resolvent estimates in Beurling-Sobolev algebras, St. Petersburg Math. J. 10 (1999), 901-964.

[4] N. K. Nikolski, In search of the invisible spectrum, Ann. Inst. Fourier (Grenoble) 49 (1999), 1925-1998.

[5] V. Pták, Extremum problems, in: Linear and Complex Analysis Problem Book 3, Part I, Lecture Notes in Math. 1573, Springer, 1994, 145-146.

[6] H. S. Shapiro, A counterexample in harmonic analysis, in: Approximation Theory, Banach Center Publ. 4, PWN (Polish Scientific Publishers), Warszawa, 1979. 
[7] Handwritten notes taken by A. Olofsson at a seminar by H. S. Shapiro entitled "Bounds for the norm of the inverse element in the Banach algebra of absolutely convergent Taylor series" held at KTH, May 23, 1997.

Department of Mathematics

Stockholm University

SE-106 91 Stockholm, Sweden

E-mail: anderso@matematik.su.se

Received July 3, 2000

Revised version January 3, 2001 